\title{
Desmoid Fibromatosis of the Right Maxilla in an Infant:
}

\section{A Case Report}

\author{
Vineet Kaul ${ }^{1}$, Biswajyoti Hazarika ${ }^{2}$, Manish Julaha ${ }^{3}$, Nandini Hazarika ${ }^{4}$, Shilpi Modi ${ }^{5}$
}

\begin{abstract}
Desmoid fibromatoses are rare benign tumors that arise from the musculoaponeurotic tissues. They are locally aggressive and invade surrounding structures. We report a case of desmoid fibromatosis of the right maxilla in a 10-month-old male baby. This site of occurrence of desmoid tumor in an infant has not been reported previously in literature. Using Weber-Ferguson incision, wide excision with right upper alveolectomy was done to remove the tumor. Adjuvant chemotherapy consisting of a weekly regimen of vinblastine and methotrexate was given to achieve locoregional control and prevent disease recurrence.

Keywords: Desmoid fibromatosis, Pediatric tumors, Spindle cell neoplasm.

Otorhinolaryngology Clinics: An International Journal (2019): 10.5005/jp-journals-10003-1321
\end{abstract}

\section{BACKGROUND}

The term desmoid was coined by Muller in 1838 which was derived from the Greek word desmos, meaning tendon-like. 7-15\% of all desmoid tumors are seen in the head and neck region, 57\% of which occur in the pediatric population. ${ }^{1}$ The incidence is estimated between two to four cases per million each year, and comprising about $0.03 \%$ of all neoplasms. ${ }^{2}$ They are aggressive tumors of mesenchymal origin, resulting from the proliferation of well-differentiated fibroblasts. ${ }^{3,4}$ At microscopy, desmoid tumors are poorly circumscribed, infiltrate the surrounding tissue, lack a true capsule and are composed of abundant collagen surrounding poorly circumscribed bundles of elongated, slender, spindleshaped cells of uniform appearance. ${ }^{5}$

\section{Case Description}

A 10-month-old male baby with a large mass protruding from his mouth was brought by his parents to the head and neck surgery clinic. The baby was active and taking liquid feeds with the help of a feeding bottle from the left side of the mouth. A detailed clinical history revealed that the baby developed a gradually progressive mass in the oral cavity for three months. On local examination, the globular mass was seen protruding out of the oral commissure along with swelling over the right cheek. The total size of the mass was $9 \mathrm{~cm} \times 6 \mathrm{~cm}$; the rest of the oral cavity could not be examined due to the mass. No neck nodes were found on clinical examination (Figs 1 to 3 ).

PET-CT showed mass present in the right maxillary region entering the oral cavity and abutting the right mandible, reaching up to infratemporal fossa and orbital floor on the right side. Blood smear examination and bone marrow study were normal. Punch biopsy revealed it to be a spindle cell tumor.

To secure the airway, tracheostomy was done under local anesthesia. Weber-Ferguson incision was given on the right side, and cheek flap elevated. Dissection of the tumor from the overlying soft tissues was done. Right upper alveolectomy was done and the tumor was excised in toto. The tumor was seen to be arising from right upper alveolus extending into the oral cavity and pushing the mucosa of the hard palate. The tumor was extending up to
${ }^{1}$ Department of ENT, Netaji Subhash Chandra Bose Medical College, Jabalpur, Madhya Pradesh, India

2,3 Department of Head and Neck Surgery, Artemis Hospital, Gurugram, Haryana, India

${ }^{4}$ Department of Medical and Paediatric Oncology, Fortis Memorial Research Institute, Gurugram, Haryana, India

${ }^{5}$ Department of Pathology, Artemis Hospital, Gurugram, Haryana, India

Corresponding Author: Vineet Kaul, Department of ENT, Netaji Subhash Chandra Bose Medical College, Jabalpur, Madhya Pradesh, India, Phone: +91 8527866560, e-mail: vineet2006@gmail.com

How to cite this article: Kaul V, Hazarika B, Julaha M, et al. Desmoid Fibromatosis of the Right Maxilla in an Infant: A Case Report. Int J Otorhinolaryngol Clin 2019;11(1):19-21.

Source of support: Nil

Conflict of interest: None

infratemporal fossa and infraorbital region from where it was excised. Skin grafting was done for the mucosal defect.

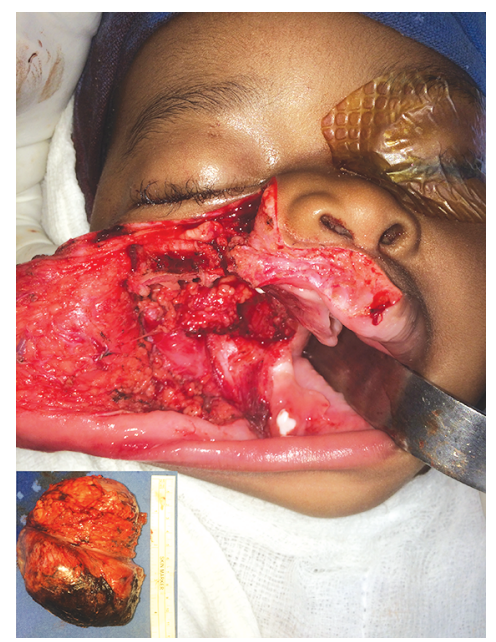

Fig. 1: Intraoperative image showing defect after tumor removal. (Inset) Globular mass measuring $9 \mathrm{~cm} \times 6 \mathrm{~cm}$ mass was removed 


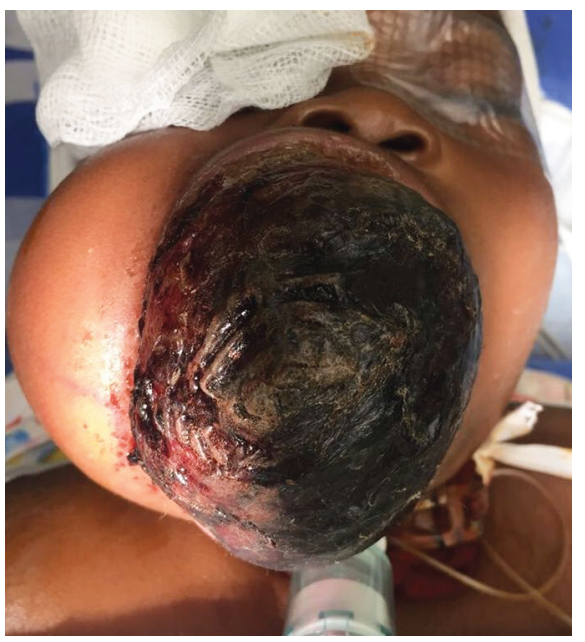

Fig. 2: Preoperative image showing mass protruding out of oral cavity with right cheek swelling

Postoperatively the baby was put on nasogastric feeds. Decannulation was done on the 6th postoperative day. Follow-up after two weeks showed good healing of the graft. The nasogastric tube was removed, and oral feeds were started, which the baby tolerated well.

Histopathological examination revealed tumor comprising of sheets and fascicles of spindle cells having spindled, focally wavy nuclei with indistinct cell boundaries along with areas of myxoid change and interspersed congested vascular spaces with scattered mitosis. These findings were consistent with the spindle cell neoplasm.

Forfurther characterization of the tumor, immunohistochemistry analysis was done which was positive for vimentin, smooth muscle actin and beta-catenin and negative for desmin, S-100, CD34, cytokeratin, h-caldesmon, and CD56. These findings were compatible with desmoid fibromatosis.

Adjuvant chemotherapy with a weekly regimen of methotrexate and vinblastine was given to preclude locoregional recurrence and the perils of revision surgery in the baby.

\section{Discussion}

Desmoid tumors are more common between the ages of 15-60 years and show a female predominance. ${ }^{6}$ According to their location, desmoid tumors are classified into intra-abdominal and extra-abdominal types. Extra-abdominal desmoid tumors comprise one-third of all desmoid tumors and usually occur in the head and neck, shoulder, pelvic girdle, and limbs. In the head and neck region, desmoid tumors are more common in the neck. To the best of our knowledge, no case of a desmoid tumor of the maxilla in an infant has ever been reported previously in literature.

Desmoid fibromatoses are benign tumors but their true pathogenic potential lies in their ability to invade surrounding structures. This makes the treatment of desmoid tumors more exigent in the head and neck region due to the dense anatomy, cosmetic pitfalls, and important neurovascular structures.

Cells typically stain diffusely for vimentin, an intermediate filament expressed in mesenchymal cells, as well as for smooth muscle actin. Tumor cells demonstrate an absence of desmin, S-100, and CD34.

Wide surgical excision is the accepted treatment modality. However, because of difficulty in achieving clear margins around

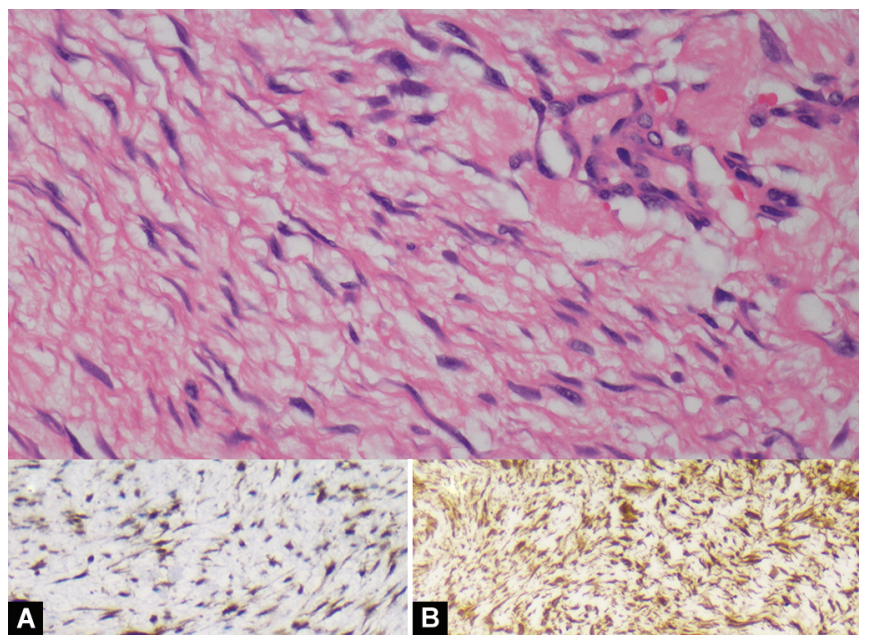

Figs 3 A and B: High power magnification (400x) showing cells having spindled, focally wavy nuclei. (Inset) Immunohistochemical study showing cells positive for beta-catenin (A) and vimentin (B)

important structures in the head and neck region, multimodality treatment is usually employed for disease control. Head and neck desmoid tumors frequently involve vital structures, and residual disease is common after resection. Faulkner et al. identified a negative margin of resection associated with the least incidence of recurrence in $70 \%$ of subjects. ${ }^{7}$ In adults, Goy et al. reported excellent control for positive margins with postoperative radiation therapy. ${ }^{8}$ This is particularly crucial if the morbidity of recurrence is high or the chances for salvage low. Radiation therapy alone has also been advocated in adults to a total dose of 55-60 Gray if the operative risk is high or cosmetic or functional morbidity is immense. ${ }^{9}$ Therefore, if a desmoid tumor is resectable with acceptable morbidity and cosmetic and functional outcome, surgery is the treatment of choice.

In children, Skapek et al. reported that combination chemotherapy with vinblastine and methotrexate achieved tumor control in half of the total subjects without significant acute or longterm morbidity. ${ }^{10}$ Combination regimens consisting of doxorubicin/ dacarbazine ${ }^{11}$ and vincristine/actinomycin/cyclophosphamide ${ }^{12,13}$ can also be given. Overall response rates for adult and pediatric patients receiving combination chemotherapy is in the range of $17-100 \%$ with a median of $50 \% .^{14}$

Non-cytotoxic drug therapy has been advocated recently in the treatment of inoperable desmoid tumors. The primary noncytotoxic treatment options are non-steroidal anti-inflammatory agents and hormonal agents. A systematic review of trials and case series of adults treated with NSAIDs found a response rate of around $50 \%$, although many responses took over 24 months to manifest. ${ }^{14}$ However, the paucity of reported cases and randomized trials relegates the validity of these results and mandates further research.

\section{Conclusion}

In conclusion, desmoid fibromatosis is a rare benign tumor with a high disposition for local recurrence. Wide excision is the mainstay of treatment with favorable long-term outcome. A negative margin of resection extenuates the incidence of locoregional recurrence. In the head and neck region, due to the exigency of obtaining negative margins, multimodality treatment is usually practiced. In the pediatric age group, this consists of adjuvant chemotherapy which is given to tackle the predicament of tumor recurrence. 


\section{References}

1. Zheng Z, Jordan AC, Hackett AM, et al. Pediatric desmoids fibromatosis of the parapharyngeal space: a case report and review of literature. Am J Otolaryngol 2016;37(4):372-375. DOI: 10.1016/j. amjoto.2016.02.003. PMID: 27040413.

2. Salman Basha M, Dutt SC, Narsimha Murthy S, et al. Juvenile fibromatosis of the temporomandibular joint: a rare case report. Dent Res J 2014;11(2):284-287.

3. Moslein G, Dozois RR. Desmoid tumors associated with familial adenomatous polyposis. Perspectives in colon and rectal. Surgery 1998;10:109-126.

4. Sagar PM, Moslein G, Dozois RR. Management of desmoids tumors in patients after ileal pouch-anal anastomosis for familial adenomatous polyposis. Dis Colon Rectum 1998;41:1350-1355. DOI: 10.1007/ BF02237046.

5. Hartley JE, Church JM, Gupta S, et al. Significance of incidental desmoids identified during surgery for familial adenomatous polyposis. Dis Colon Rectum 2004;47(3):334-338. DOI: 10.1007/ s10350-003-0063-0.

6. Shih HA, Hornicek FJ, DeLaney TF. Fibromatosis: current strategies for treatment.Curr Opin Orthop 2003;14:405-412. DOI: 10.1097/00001433200312000-00009.

7. Faulkner LB, Hajdu SI, Kher U, et al. Pediatric desmoid tumor: a retrospective analysis of 63 cases. J Clin Oncol 1995;13(11):2813-2818. DOI: $10.1200 / J C O .1995 .13 .11 .2813$.
8. Goy BW, Lee SP, Eilber F. The role of adjuvant radiotherapy in the treatment of resectable desmoids tumors. Int J Radiat Oncol Biol Phys 1997;39(3):659-665. DOI: 10.1016/S0360-3016(97) 00334-9.

9. Anderson T, Weinstein GS, Harwick J, et al. Hypopharyngeal desmoid tumor. Otolaryngology Head Neck Surg 2000;123(3):279-281. DOI: 10.1067/mhn.2000.104521.

10. Skapek SX, Hawk BJ, Hoffer FA, et al. Combination chemotherapy using vinblastine and methotrexate for the treatment of progressive desmoid tumor in children. J Clin Oncol 1998;16(9):3021-3027. DOI: 10.1200/JCO.1998.16.9.3021. PMID: 9738571.

11. Goepfert H, Cangir A, Ayala AG, et al. Chemotherapy of locally aggressive head and neck tumors in the pediatric age group. Desmoid fibromatosis and nasopharyngeal angiofibroma. Am J Surg 1982;144(4):437-444. DOI: 10.1016/0002-9610(82) 90418-4.

12. Raney B, Evans A, Granowetter L, et al. Nonsurgical management of children with recurrent or unresectable fibromatosis. Pediatrics 1987;79(3):394-398.

13. Raney RB. Chemotherapy for children with aggressive fibromatosis and Langerhans' cell histiocytosis. Clin Orthop Relat Res 1991(262):58. DOI: 10.1097/00003086-199101000-00008.

14. Janinis J, Patriki M, Vini L, et al. The pharmacological treatment of aggressive fibromatosis: a systematic review. Ann Oncol 2003;14(2):181-190. DOI: 10.1093/annonc/mdg064. 